\title{
« Les Irlandais attendent avec une légère impatience » : Paris et la demande d'adhésion de Dublin à la CEE, 1961-1973
}

\section{Christophe Gillissen}

\section{(2) OpenEdition \\ 1 Journals}

\section{Édition électronique}

URL : http://journals.openedition.org/etudesirlandaises/2929

DOI : 10.4000/etudesirlandaises.2929

ISSN : 2259-8863

\section{Éditeur}

Presses universitaires de Rennes

\section{Édition imprimée}

Date de publication : 30 juin 2012

Pagination : 9-23

ISSN : 0183-973X

\section{Référence électronique}

Christophe Gillissen, « « Les Irlandais attendent avec une légère impatience » : Paris et la demande d'adhésion de Dublin à la CEE, 1961-1973 », Études irlandaises [En ligne], 37-1 | 2012, mis en ligne le 30 juin 2014, consulté le 28 avril 2019. URL : http://journals.openedition.org/etudesirlandaises/2929; DOI : 10.4000/etudesirlandaises.2929 


\title{
"Les Irlandais attendent avec une légère impatience " : Paris et la demande d'adhésion de Dublin à la CEE, 1961-1973
}

\author{
Christophe Gillissen \\ Université de Paris 4 - Sorbonne
}

\section{Résumé}

La première demande d'adhésion irlandaise à la CEE, en 1961, suscita une forte réserve de la part de Paris. Malgré les réticences des cinq autres Etats membres, la France bloqua toute décision sur la demande irlandaise jusqu'en octobre 1962. En revanche, Paris se montra nettement plus favorable à la candidature irlandaise lorsqu'elle fut réactivée en 1967. Ce revirement de la politique française s'explique en partie par la diplomatie irlandaise qui sut faire valoir auprès des autorités françaises qu'elles avaient tout à gagner de l'entrée de l'Irlande dans la CEE.

Mots clé : Irlande, France, Grande-Bretagne, CEE, Seán Lemass, Charles de Gaulle.

\section{Abstract}

The first Irish application for EEC membership in 1961 met with considerable hostility from Paris. Despite the reluctance of the five other member states, France postponed a decision on the Irish application until October 1962. However, when Ireland reactivated its application in 1967, the attitude of Paris proved much more favourable. Such a change in French policy was partly due to Irish diplomacy which convinced French authorities they had much to gain from Ireland's admission to the EEC.

Keywords: Ireland, France, Great Britain, EEC, Seán Lemass, Charles de Gaulle.

Seán Lemass, Premier ministre irlandais de 1959 à 1967, était convaincu qu’il fallait ouvrir l'économie de son pays au commerce international afin de relancer la croissance et créer des emplois. À cette fin, il avait envisagé une adhésion à la Communauté économique européenne (CEE) dès le début de 1959, alors qu’il n'était encore que ministre de l'Industrie et du commerce : il avait dit au secrétaire du Conseil irlandais du Mouvement européen que «le temps était venu de réfléchir sérieusement à l'Europe car il n'était plus exclu que l'Irlande demande à devenir membre à part entière de la $\mathrm{CEE}^{1}$ ". Il semble avoir évoqué cette possibilité avec son homologue britannique, Reginald Maudling, le 26 mai 1959, lors de

1. Denis Corboy, «Journey to Europe, first shoots : a personal memoir », p. 60, in Jane Conroy (dir.), Franco-Irish Connections, Dublin, Four Courts Press, 2009, p. 57-64. 
discussions au sujet des projets européens de libre-échange et de "leurs implications pour le commerce anglo-irlandais ${ }^{2}$ ".

La réaction britannique ne fut guère encourageante : Lemass expliqua plus tard aux Français que "Londres s'était opposé, en menaçant de dénoncer le Traité de commerce anglo-irlandais, à ce que l'Eire demande à adhérer au marché commun $^{3}$ ". Mais lorsque le gouvernement britannique modifia sa propre position vis-à-vis de l'Europe, rien ne s'opposait plus à ce que Dublin déposât une demande officielle d'adhésion, ce qui fut fait le 31 juillet 1961. La demande britannique suivit quelques jours plus tard, le 10 août.

Dès le 8 novembre 1961, le Conseil européen se prononça en faveur de l'ouverture de négociations avec Londres; en revanche, il temporisa vis-à-vis de Dublin et ne donna son aval que le 23 octobre $1962^{4}$. Ainsi, pendant près d'un an, l'Irlande se retrouva-t-elle dans la situation pour le moins inconfortable de devoir envisager la possibilité que son principal partenaire commercial - $75 \%$ des exportations irlandaises étaient alors vendues sur le marché britannique - accédât à une union douanière dont elle-même serait exclue. Ce délai fut dû à la France, qui s'opposa à la candidature irlandaise de manière systématique jusqu'au Conseil européen du 23 octobre 1962.

Afin de comprendre les raisons de ce refus et la manière dont l'Irlande obtint gain de cause au bout du compte, cet article, qui s'appuie en particulier sur les archives françaises, suivra les principales étapes des contacts entre Paris et Dublin de 1961 à 1973.

\section{圈 L'hostilité initiale de Paris}

Plusieurs éléments permettent de comprendre les raisons de l'hostilité initiale de Paris à l'égard de la demande irlandaise. Tout d'abord, l'Irlande n'était pas arrivée à un stade de développement suffisant pour qu'elle puisse aisément s'intégrer au Marché commun selon un rapport français, qui notait que le produit national du pays était "l'un des plus bas d'Europe ", tout comme son taux de croissance 5 . Il soulignait en outre le " protectionnisme massif » du régime douanier irlandais, ainsi que sa " réglementation phytosanitaire aussi étendue et plus fantaisiste que l'anglaise ». Des efforts considérables seraient donc nécessaires pour s'adapter aux exigences du Marché commun, mais rien ne laissait penser que l'in-

2. Dáil debates, vol. 175, col. 585 (27 mai 1959).

3. Affaires étrangères - Archives diplomatiques, La Courneuve (ci-après AEAD) : Secrétariat général, vol. 17, f. 79 (P 11921) : "Conversations franco-irlandaises ", 13 oct. 1962.

4. D. J. Maher, The Tortuous Path: The course of Ireland's entry into the EEC, 1948-73, Dublin, Institute of Public Administration, 1986, p. 138.

5. Archives diplomatiques, Nantes (ci-après AD) : Dublin, vol. 220, n 2/CE : « Note : Position économique de l'Irlande ", 15 jan. 1962. 
dustrie irlandaise serait en mesure de supporter le poids de la concurrence européenne. Paris s'étonnait d'ailleurs que la formule d'adhésion avait été retenue, puisqu'une formule d'association, a priori plus adaptée au cas de l'Irlande, aurait suffi à préserver ses échanges commerciaux avec la Grande-Bretagne ${ }^{6}$.

Un autre problème soulevé par la candidature irlandaise était la politique étrangère de Dublin, dont la neutralité ne correspondait guère aux objectifs politiques des $\mathrm{Six}^{7}$. La délégation irlandaise aux Nations unies prenait alors une part active aux efforts pour accélérer la décolonisation et pour freiner la course aux armements, ce qui la mettait en porte-à-faux avec certains Etats membres de la CEE. Pour Paris, l'affiliation de l'Irlande "à un groupe régional de nature politico-économique " paraissait "à première vue difficilement compatible avec l'orientation de sa politique extérieure ${ }^{8}$ ".

Enfin, la candidature irlandaise soulevait la question de l'élargissement de la CEE. L'admission de quatre nouveaux membres - outre l'Irlande et la GrandeBretagne, le Danemark et la Norvège étaient aussi candidats - porterait atteinte au statut du français. En effet, si l'usage voulait que les négociations fussent menées en français, les Irlandais et les Danois firent savoir que leurs délégations s'exprimeraient en anglais. De ce point de vue, les négociations d'adhésion marquèrent « le début d'une évolution " néfaste pour le français? .

Plus généralement, les autorités françaises pensaient que l'élargissement de la CEE en modifierait profondément la nature, au point de compromettre le projet d'intégration européenne :

Il est évident qu'un marché commun à sept ou à dix ne ressemblera en rien au marché commun primitif. L'équilibre politique y sera modifié du tout au tout. La primauté de l'influence française ne pourra être maintenue. À la conjonction franco-allemande qui a été jusqu'à présent pratiquée se substituera au mieux une rivalité franco-anglaise. Sur bien des questions, l'Allemagne se ralliera aux thèses anglaises. La tentative de construction d'une Europe politique à peine ébauchée, ne pourra être poursuivie, faute de buts communs ${ }^{10}$.

6. AEAD : Coopération économique, vol. 1391, f. 127 : « Note : Réunion du 18 janvier avec la délégation irlandaise », 13 jan. 1962.

7. Dermot Keogh, "Irish Neutrality and the First Application for Membership of the EEC, 1961-3 ", in Michael Kennedy et Joseph Morrison Skelly (dir.), Irish Foreign Policy 1919-1966, Dublin, Four Courts Press, 2000, p. 265-285.

8. AEAD : Coopération économique, vol. 1391, f. 127 : « Note : Réunion du 18 janvier avec la délégation irlandaise ", 13 jan. 1962.

9. AEAD : Archives orales, vol. 19, Boegner, $\mathrm{n}^{\circ}$ 3.1.3.

10. AEAD : Affaires financières et économiques, Direction économique, vol. 47 : "Note a.s. adhésion de l'Angleterre au marché commun ", 28 août 1961. 
On le voit, pour Paris le problème de l'élargissement tenait avant tout à la candidature de Londres. Plusieurs réunions furent tenues à l'Elysée par le général de Gaulle en présence de quelques ministres et hauts fonctionnaires, afin de définir une position sur la demande britannique. De leur point de vue, elle transformerait le Marché commun en " une vaste communauté atlantique fondée sur le principe du libre-échange ${ }^{11}$ ". Si cela n'était pas acceptable, il fallait cependant faire preuve de prudence, car la candidature britannique était soutenue par les cinq autres membres de la CEE et une rupture brutale des négociations pourrait compromettre " la suite de la politique agricole commune ${ }^{12}$ ". Il fut donc convenu de "faire perdre la négociation dans les sables " en demandant au gouvernement britannique des concessions sur l'agriculture, dans l'espoir qu'il les refuse et ainsi endosse lui-même la responsabilité de l'échec des négociations.

En d'autres termes, Paris cherchait à gagner du temps, et la candidature irlandaise, dont les faiblesses étaient manifestes, se prêtait bien à une stratégie dilatoire. Une note interne du Quai d'Orsay, datée du mois d'octobre 1962, confirme que c'était la raison principale de l'hostilité française contre la candidature de Dublin : "Jusqu'ici nous nous sommes refusés à accepter la "recevabilité" de la demande irlandaise, étant donné les inconvénients que nous voyons à l'élargissement du Marché en une Communauté de 8 à 10 pays $^{13}$. "

\section{Le lobbying de Dublin}

Par leurs contacts au sein de la CEE, les autorités irlandaises avaient vite compris que leurs difficultés étaient dues pour l'essentiel à la France ${ }^{14}$. Il leur fallait donc dissiper tout malentendu quant à leur demande d'adhésion auprès des autorités françaises. Dès le mois d'août 1961, l'ambassadeur irlandais rencontra le ministre français des Affaires étrangères, Maurice Couve de Murville, et précisa que l'entrée de l'Irlande ne reviendrait pas à " un vote supplémentaire » pour Londres dans les instances européennes ${ }^{15}$. Non seulement l'Irlande n'était pas un satellite de la Grande-Bretagne, mais il n'était pas exclu que dans certains domaines, comme la politique agricole, Dublin votât avec Paris.

11. AN : 5 AG 1, Elysée, vol. 24 : "Compte rendu du Conseil restreint relatif aux Affaires étrangères, 24 mai $1962 »$.

12. AN : 5 AG 1, Elysée, vol. 24 : "Compte rendu du Conseil restreint relatif aux Affaires étrangères, 31 juillet $1962 "$.

13. AEAD : Coopération économique, vol. 1392, f. 118 : "Monsieur Wormser, de la part de J. Grunewald ", 10 oct. 1962.

14. Dermot et Aoife Keogh, "Ireland and European Integration : From the Treaty of Rome to Membership ", p. 27, in Mark Callanan (dir.), Foundations of an Ever Closer Union: An Irish perspective on the fifty years since the Treaty of Rome, Dublin, Institute of Public Administration, 2007, p. 6-50.

15. National Archives, Dublin : Department of Foreign Affairs (ci-après NA, DFA), Embassy series, Rome, 19/09/ RI : McDonald, 24 août 1961. 
Quelques jours plus tard, deux hauts fonctionnaires irlandais, T. K. Whitaker du ministère des Finances et Con Cremin des Affaires extérieures, se rendirent au Quai d'Orsay pour y rencontrer l'influent directeur des Affaires économiques, Olivier Wormser. Ils lui expliquèrent que leur pays n'était neutre «ni juridiquement, ni idéologiquement ", qu'il n'entretenait pas de relations avec les pays communistes, et que sa présence dans la CEE ne compromettrait pas "l'homogénéité de ce groupement ${ }^{16}$ ".

Pour sa part, le Taoiseach (Premier ministre), Lemass, multiplia les déclarations iconoclastes sur la neutralité irlandaise, affirmant que son pays participerait pleinement aux projets d'intégration politique de la CEE, y compris dans leur dimension militaire ${ }^{17}$. Il fut même envisagé de mettre à la disposition des États-Unis une base aéronavale dans l'estuaire du Shannon, afin de désarmer l'opposition de ceux qui considéraient « le maintien par l'Irlande d'une attitude officielle de neutralité comme incompatible avec son admission" dans la $\mathrm{CEE}^{18}$.

Mais ces contacts et déclarations ne suffirent pas à débloquer la situation : plus d'un an après la candidature irlandaise, le Conseil des ministres de l'Europe n'avait toujours pas pris de décision, en raison de l'obstruction de Paris. En effet, les autorités françaises indiquèrent qu'elles en étaient encore à l'étude de la recevabilité de cette candidature, tout en étant conscientes que Dublin attendait " avec une légère impatience » une réponse à sa demande d'ouverture de négociations ${ }^{19}$.

Lemass décida alors de prendre l'initiative : en octobre 1962, il fit une tournée des capitales des Six pour y rencontrer les chefs d'Etat et de gouvernement ${ }^{20}$. La veille de son arrivée à Paris, le Quai d'Orsay transmit au Président et au ministre des Affaires étrangères, qui devaient s'entretenir avec lui, quelques indications visant à expliquer la position de la France :

Ce n'est pas par négligence que nous avons tardé à répondre à la demande irlandaise. Nous sommes très conscients de l'importance du problème pour l'Irlande. Mais en toute honnêteté il nous paraît difficile de fournir dès maintenant une réponse tant soit peu concrète. Une des données du problème nous parait être en effet que l'Irlande a intérêt à se trouver du même côté que la Grande-Bretagne par rapport au tarif extérieur commun de la CEE. Si tel est bien le cas, il n'est guère possible de définir des solutions $[\ldots]$ tant que l'entrée de l'Angleterre n'est pas décidée $[. .$.$] .$

16. AEAD : Coopération économique, vol. 1391, f. 38 : Wormser, 7 sept. 1961.

17. Michael J. Geary, An Inconvenient Wait : Ireland's Quest for Membership of the EEC, 1957-73, Dublin, Institute of Public Administration, 2009, p. 39-45.

18. AEAD : Coopération économique, Vol. 1391, f. 180 : Paris, 19 fév. 1962.

19. AEAD : Europe, Irlande, vol. 46 : « Note : a.s. L'Irlande. Politique extérieure », 10 oct. 1962.

20. Maurice FitzGerald, Protectionism and Liberalisation : Ireland and the EEC, 1957-1966, Aldershot, Ashgate, 2000, p. 192-200. 
Ce que nous pouvons dire néanmoins c'est que nous étudierons le cas de l'Irlande avec la plus grande bienveillance et que, si l'Angleterre entre dans la Communauté, nous chercherons une formule qui permette à l'Irlande d'être du même côté que l'Angleterre par rapport au tarif extérieur commun ${ }^{21}$.

Pour Paris, il n'était donc pas question à ce stade d'accepter la demande d'adhésion de Dublin.

\section{圈 L'entretien du général de Gaulle avec Lemass}

Au début de son entretien avec le général de Gaulle, Lemass l'informa du désir qu'éprouvait son pays « à voir le Conseil des Ministres de la Communauté Européenne prendre une décision favorable quant à l'ouverture de négociations avec son pays ${ }^{22}$ ". Il ne demandait pas que de réelles négociations fussent entamées tant que les pourparlers avec la Grande-Bretagne étaient en cours, mais il fallait que Dublin pût «prouver à son opinion publique la nécessité de prendre certaines mesures dès maintenant en vue d'adapter l'économie du pays aux nécessités au Marché commun ».

De Gaulle, après avoir évoqué la dépendance irlandaise vis-à-vis de la GrandeBretagne, pria « son interlocuteur de lui expliquer quelles seraient les mesures que prendrait l'Irlande dans l'hypothèse où la Grande-Bretagne deviendrait membre du Marché Commun. " Le Taoiseach lui répondit que l'Irlande était convaincue de pouvoir "s'adapter à un marché plus large " et qu'elle en tirerait des bénéfices, tant du point de vue de son secteur industriel que de son secteur agricole; elle avait donc "l'intention de s'engager sans réserve dans un marché commun économique».

Le Président aborda ensuite la dimension politique, rappelant à Lemass que les Six s'étaient unis en partie pour faire face à la menace soviétique, et que si " la constitution politique de l'Europe ne devait pas se faire, le Marché Commun lui-même tendrait à se disloquer. " Dans ce contexte, il interrogea Lemass quant à la position de l'Irlande, " pays insulaire qui [n'avait] point partagé avec les pays continentaux l'épreuve de la dernière guerre, face à une intégration politique éventuelle d'une association qui ne [pouvait] pas et ne [voulait] pas demeurer neutre».

21. AEAD : Europe, Irlande, vol. 46 : «Voyage de M. Lemass », 12 oct. 1962.

22. Archives nationales, Paris (ci-après AN) : 5 AG 1, Elysée, vol. 177 : «Entretien du Général de Gaulle avec M. Lemass, Premier ministre d'Irlande, 13 octobre 1962, à 12h30 " (ce document, pour lequel il n'y a pas d'équivalent dans les archives irlandaises, est cité abondamment dans la mesure où jusqu'à présent les publications sur l'adhésion irlandaise à la CEE ont dû se contenter de sources indirectes pour cerner la teneur de l'entretien). 
Le Premier ministre irlandais assura son interlocuteur que son pays acceptait "sans aucune réserve toutes les conséquences politiques de l'adhésion éventuelle de l'Irlande au Marché Commun ", ajoutant que la candidature irlandaise était " motivée peut-être plus par les considérations politiques que par les avantages économiques ". Il précisa que l'Irlande savait parfaitement que la CEE était un premier pas qui mènerait "sans le moindre doute à une organisation politique " et qu'il était évident que toute organisation européenne s'inspirant d'un même objectif politique devait " également avoir une politique commune de défense à laquelle l'Irlande participerait pleinement ». Il continua :

L'Irlande accueillerait favorablement l'occasion historique d'une participation à une organisation politique européenne, ne serait-ce que pour prouver sans ambiguïté qu'elle se refuse de prendre une position neutraliste dans le monde. Le peuple irlandais est conscient de son identité européenne, ne fût-ce que par les liens historiques qui l'unissent aux pays continentaux catholiques.

Lemass rencontra ensuite le ministre des Affaires étrangères, Maurice Couve de Murville, qui lui indiqua que «la France ne ferait pas opposition, au prochain Conseil de Bruxelles, à la demande irlandaise d'adhésion ", ce dont l'Irlandais se montra " fort satisfait ${ }^{23}$ ".

En apparence, les efforts de Lemass avaient été couronnés de succès, mais le geste de Paris n'était qu'une concession formelle qui ne changeait rien à sa position de fond. En effet, si le Conseil européen du 23 octobre 1962 donna son aval à l'ouverture de négociations avec l'Irlande, Paris insista pour qu'il fût clairement précisé qu'elles ne pouvaient débuter dans l'immédiat et que c'était aux Six que revenait « le droit d'apprécier si l'adhésion " constituait " en définitive la solution la plus appropriée » pour l'Irlande ${ }^{24}$.

En somme, les autorités françaises cherchaient toujours à faire obstruction à l'élargissement de la CEE, mais face à ces atermoiements, Bruxelles et La Haye en vinrent à menacer de bloquer les dossiers en cours - notamment la politique agricole commune - tant que la question britannique ne serait pas réglée. Le général de Gaulle, d'une "humeur épouvantable ", décida alors de trancher dans le vif ${ }^{25}$ : il tint sa célèbre conférence de presse du 14 janvier 1963 où il opposa son veto à la demande d'adhésion de Londres, mettant un terme par la même occasion à la candidature irlandaise.

23. AEAD : Secrétariat général, vol. 17, fo 79 (P 11921) : "Conversation avec le Premier Ministre d'Irlande ", 13 oct. 1962.

24. AEAD : Coopération économique, vol. 1392, f 116-117 : "Note : Négociations entre la CEE et l'Irlande ", 20 oct. 1962.

25. AEAD : Archives orales, vol. 26, Lucet, $n^{\circ} 4.1 .5$. 


\section{Visites officielles et accord culturel}

Dublin ne se découragea pas pour autant. L'objectif d'une adhésion fut maintenu, même s'il fallut repousser l'échéance : pour Lemass, le but était désormais d'accéder à la CEE avant $1970^{26}$. Dans cette perspective, un bilan de la candidature initiale montrait à quel point il était important, sinon vital, de s'assurer du soutien de Paris. La diplomatie irlandaise multiplia donc les visites officielles, afin de nouer des relations d'amitié avec les décideurs français et de se constituer ainsi un capital de sympathie avec la principale puissance de la CEE.

En novembre 1963, Frank Aiken, ministre des Affaires extérieures, rencontra Charles de Gaulle à Paris, pour discuter notamment de la candidature irlandaise. Le Président lui dit comprendre "le désir de l'Irlande de s'associer au Marché commun » et affirma que "la France n'y serait pas opposée $e^{27}$ ». Il expliqua que, "pour beaucoup de raisons, politiques, économiques et historiques", son pays était favorable à l'Irlande, "ainsi qu'à l'entrée de celle-ci dans l'ensemble européen ". Aiken répondit que l'Irlande, " après avoir été coupée de l'Europe par la Grande-Bretagne pendant sept cents ans ", désirait, pour des raisons historiques aussi, être associée à l'Europe. Aiken profita de l'entretien pour inviter le Général à se rendre en Irlande, insistant "sur le plaisir qu'éprouveraient le gouvernement et le peuple irlandais » à le recevoir.

Si le Président ne donna pas suite à cette invitation, une visite officielle de grande importance eut lieu en juin 1964 lorsque, pour la première fois depuis la fondation de l'Etat irlandais, un ministre français des Affaires étrangères se rendit à Dublin. Couve de Murville s'y entretint avec plusieurs ministres, ainsi qu'avec le Président, Eamon de Valera. Les discussions, qui portèrent sur le commerce franco-irlandais, la politique internationale et la CEE, eurent lieu dans un «climat de cordialité et de confiance mutuelle "selon le communiqué final ${ }^{28}$. Sur la question des candidatures irlandaise et britannique à la CEE, Couve déclara que « le problème pour l'Angleterre est de savoir si elle se sent ou non européenne. Aucun problème de ce genre ne se pose pour l'Irlande qui, elle, a cette conviction ${ }^{29}$ ".

Ce changement de ton dans les relations franco-irlandaises s'explique en partie par la diplomatie irlandaise. Outre l'accueil "particulièrement cordial " réservé au ministre selon une note française, la proposition de jeter les bases d'un accord culturel bilatéral fut également fort bien reçue par Paris. Il s'agissait de promou-

26. AEAD : Europe, Irlande, vol. 49, nº 106/CE : « Note : a.s. Irlande et CEE », 4 juin 1964.

27. AEAD : Cabinet du Ministre, Couve de Murville (1963), Entretiens et messages : «Entretien du Général de Gaulle et de M. Aiken, Ministre des Affaires étrangères d'Irlande, le mercredi 20 novembre 1963, de 16h 30 à $17 \mathrm{~h} 15$.»

28. AEAD : Europe, Irlande, vol. 49, 12 juin 1964.

29. AEAD : Europe, Irlande, vol. 49, «Entretien entre le Premier ministre d'Irlande et M. Couve de Murville ", 11 juin 1964. 
voir l'enseignement du français dans les écoles et universités irlandaises, et de pourvoir à la formation des enseignants de français en Irlande, mesures qui ne pouvaient que consolider le statut du français dans une CEE élargie. L'ambassadeur français à Dublin, rappelant que l'Irlande n'avait signé d'accord culturel qu'avec la France, nota : "Si l'adhésion éventuelle de l'Irlande signifie qu'un pays anglophone va entrer dans la Communauté, ce pays manifeste pour notre langue et notre culture le plus grand intérêt ${ }^{30}$. " Le fait que la délégation irlandaise à la Commission culturelle franco-irlandaise fût dirigée par le secrétaire général du ministère des Affaires étrangères en disait " long à cet égard » selon lui.

En somme, les efforts de Dublin amenèrent Paris à voir sa demande d'adhésion sous un jour nouveau et à conclure qu'elle n'était pas de nature à menacer les intérêts français, au contraire. De ce point de vue, la visite de Couve de Murville marqua sans aucun doute un tournant dans les relations entre les deux pays. Le Quai d'Orsay estima pour sa part qu'elle avait «certainement contribué à resserrer des liens anciens que l'écran de la Grande-Bretagne avait déjà depuis longtemps notablement distendu ${ }^{31}$ ».

\section{圈 Deuxième tentative}

De fait, lorsque Dublin réactiva sa candidature en mai 1967, les réactions de Paris furent tout autres qu'en 1961, témoignant ainsi d'un changement remarquable dans leurs relations bilatérales. En effet, autant l'économie irlandaise avait pu susciter des doutes sérieux au début de la décennie, autant elle ne constituait plus un obstacle en 1967. Un rapport français soulignait les progrès effectués depuis 1961, notamment suite à l'accord de libre-échange signé avec la Grande-Bretagne en 1965 : il estimait que le pays n'avait "plus guère à redouter la concurrence des pays industrialisés du Continent ${ }^{32}$ ». Quant à son agriculture, elle bénéficierait de la participation au Marché commun et de la Politique agricole commune. Enfin, en raison du poids limité de son économie, la présence de l'Irlande ne serait "guère sensible " au sein de la CEE. En conclusion, le rapport notait que sa candidature au Marché commun ne semblait " pas poser de problèmes particulièrement difficiles ".

De même, la politique extérieure de l'Irlande ne constituait plus une entrave à la recevabilité de sa demande d'adhésion. Les accords d'Evian avaient mis fin aux divergences franco-irlandaises sur l'Algérie aux Nations unies, tandis que le retrait des forces françaises du commandement intégré de l'OTAN en 1966 avait diminué l'importance de la neutralité irlandaise. Une note du Quai d'Orsay pré- 
cisait malgré tout que le pays était prêt à adhérer à une construction politique qui pouvait "déboucher sur une coopération militaire ${ }^{33}$ ». De manière générale, les relations bilatérales entre Paris et Dublin étaient perçues comme "aussi bonnes que possibles ", la France étant, parmi les "pays du continent européen, celui avec lequel le peuple irlandais se sent le plus d'affinités ${ }^{34}$ ».

Enfin, sur la question du statut du français, le gouvernement irlandais avait pris des mesures pour rassurer Paris quant aux conséquences de l'élargissement, notamment l'accord culturel franco-irlandais signé le 4 novembre 1967.

Cela étant, si Paris n'était plus opposé à la candidature irlandaise, il n'en demeurait pas moins qu'elle était toujours aussi tributaire de la demande britannique, qui avait aussi été réactivée. Or les Six étaient alors engagés dans des négociations « longues et difficiles » sur le règlement financier de la politique agricole $^{35}$. Dans ce contexte, la candidature de Londres, qui exprimait «souvent la thèse " selon laquelle ce règlement devait être renégocié ou refait, s'avérait pour le moins inopportune du point de vue français ${ }^{36}$. Se posaient aussi les questions de la livre sterling et de son statut de monnaie de réserve, de l'état de l'économie britannique, et des échanges commerciaux entre la Grande-Bretagne et les pays du Commonwealth, qui rendaient son accession à la CEE malaisée.

En octobre 1967, le général de Gaulle réunit un conseil restreint pour décider de la position à adopter sur la demande britannique. Il fut convenu que la Grande-Bretagne n'était toujours "pas mûre pour accéder au Marché commun qui, de son côté, [n'était] pas prêt à la recevoir ", et qu'il fallait donc s'opposer à nouveau à la candidature de Londres ${ }^{37}$.

Deux semaines plus tard, le Premier ministre irlandais, Jack Lynch, se rendit à Paris avec plusieurs ministres et hauts fonctionnaires pour s'entretenir des candidatures irlandaise et britannique. Les Français ne leur cachèrent pas leur position, tout en faisant preuve de compréhension quant à la situation de Dublin. Ainsi, le Président français déclara-t-il :

De la part de la France, il n'y a eu de tout temps et il n'y a aujourd'hui que de la sympathie à l'égard de l'Irlande. [...] Du point de vue de l'Europe, maintenant, il n'y aurait pas grande difficulté à ce que l'Irlande entrât dans la Communauté [...] Néanmoins, ainsi que vous le dites vousmême, la difficulté provient de vos liens étroits avec la Grande-Bretagne.

33. AEAD : Europe, Irlande, vol. 46 : « Note : Politique extérieure irlandaise », 27 oct. 1967.

34. AEAD : Europe, Irlande, vol. 49 : « Note : Relations franco-irlandaises », 27 oct. 1967.

35. AN : 5 AG 1, Elysée, vol. 48 : « Note au sujet des conséquences d’une crise possible entre les Six », 17 oct. 1967.

36. AN : 5 AG 1, Elysée, vol. 48 : "Note à l'attention du Général de Gaulle : Conditions de mise en œuvre du règlement financier à partir de 1970 », 20 oct. 1967.

37. AN : 5 AG 1, Elysée, vol. 24 : «Compte rendu du Conseil restreint relatif aux Affaires étrangères, 16 octobre 1967 ". 
[...] Dans sa situation présente, la Grande-Bretagne ne peut pas entrer dans la Communauté et elle ne le pourra pas tant qu'elle restera ce qu'elle est. Je ne sais si un jour sa situation sera différente ${ }^{38}$.

La situation avait changé du tout au tout, la France n'étant plus opposée à la candidature irlandaise; mais le problème de fond, la réticence de Paris à valider l'élargissement, était toujours là. Il n'était plus question de refuser l'adhésion de l'Irlande à la CEE, mais elle ne pourrait y accéder que lorsque la Grande-Bretagne serait elle-même admise.

\section{圈 Rapprochement}

En 1969, la démission du général de Gaulle ouvrit la perspective d'une admission rapide des pays candidats, son successeur à la présidence, Georges Pompidou étant favorable à l'élargissement de la CEE. La décision du général de Gaulle de séjourner en Irlande contribua aussi à améliorer les relations bilatérales entre Paris et Dublin ${ }^{39}$.

Il s'agissait d'une visite faite à titre privé, mais l'accueil qui lui fut réservé par les autorités irlandaises conféra à son séjour un caractère quasi officiel ${ }^{40}$. Lorsque le président, Eamon de Valera, l'invita au palais présidentiel, ils eurent l'occasion d'aborder la question européenne :

- "Vous n'êtes pas des continentaux », observa le Général de Gaulle.

- « Nous voulons l'être, répondit le Président. La Grande-Bretagne (le mot "ennemi" a été prononcé) est un écran entre l'Europe et nous ${ }^{41}$."

Avant son départ, le Premier ministre, Jack Lynch, lui offrit un déjeuner de gala au château de Dublin. À l'issue du repas, il évoqua longuement les relations franco-irlandaises, soulignant la dette de l'Irlande vis-à-vis de la France. De Gaulle prit la parole à son tour, revenant à l'idée d'un écran entre les deux pays :

Il y a, semble-t-il, eu depuis quelques générations une sorte d'écran entre l'Irlande et la France. Mais il parait que ce temps-là est passé et qu'il nous est possible à vous et à nous de le traverser, de nous trouver, d'être ensemble par l'esprit et par l'action. C'est l'impression que j'ai recueillie de mon rapide séjour et c'est le souhait que je veux former : puissent

38. AN : 5 AG 1, Elysée, vol. 177 : «Entretien du Général de Gaulle et de M. John Lynch, 3 novembre 1967, 12 h 15-13h 15 " (le prénom officiel de Lynch était John, même si en Irlande on ne l'appelait que Jack).

39. Pierre Joannon, L'Hiver du Connétable : Charles de Gaulle et l'Irlande, La Gacilly, Artus, 1991.

40. Françoise Parturier (La lettre d'Irlande, Paris, Albin Michel, 1979) donne nombre d'anecdotes révélatrices du soin pris par les autorités irlandaises pour garantir le succès du séjour de leur hôte.

41. AEAD : Europe, Irlande, vol. 52, n 302/CM : Harcourt, "a/a séjour en Irlande du Général de Gaulle, 10 mai19 juin 1969 ", 9 juil. 1969. 
l'Irlande et la France se connaître encore mieux tous les jours, se rencontrer et être ensemble dans la pensée et l'action!

Il se tourna alors vers l'assemblée, levant son verre en l'honneur de «l'Irlande toute entière " ${ }^{42}$.

Tout cela eut un impact considérable sur les opinions publiques française et irlandaise. Du côté irlandais, la visite de de Gaulle fut perçue comme une manifestation de bonne volonté fort appréciable, contribuant ainsi à amoindrir " l'amertume certaine " laissée par l'attitude restrictive de la France à l'égard de l'élargissement de la $\mathrm{CEE}^{43}$. Du côté français, grâce à la couverture médiatique de "l'exil irlandais " du général, nombre de personnes découvrirent un pays encore trop méconnu.

Ce fut en tout cas le constat d'une délégation de la Commission des Affaires étrangères de l'Assemblée nationale, qui se rendit en Irlande un mois plus tard. Son rapport, très favorable à la candidature irlandaise, nota que la France avait " un intérêt politique certain à voir adhérer l'Irlande à la CEE » et conclut qu'il fallait resserrer les liens entre Paris et Dublin :

Nous avons assisté en quelque sorte à des "retrouvailles " avec les Irlandais. Ce pays, qu'une forte personnalité différencie de la GrandeBretagne à laquelle on l'assimile trop aisément, ne demande qu'à élargir ses échanges, à renouer des contacts devenus rares avec le continent européen, et tout particulièrement avec la France.

Cette situation confère à la candidature irlandaise au Marché commun une grande importance et exige que nous développions nos relations avec ce pays dans tous les domaines. [...] C'est pourquoi nous ne saurions trop conseiller la multiplication des contacts et des échanges. L'Irlande doit redevenir familière aux Français ${ }^{44}$.

Du point de vue de Paris, il était désormais acquis que l'Irlande serait un allié dans une Communauté élargie, et il convenait de cultiver les relations francoirlandaises afin de maintenir l'influence de la France au sein de la $\mathrm{CEE}^{45}$. Ainsi, lorsque le Premier ministre irlandais rencontra le président Pompidou à Paris en 1972, ils se mirent d'accord sur plusieurs points, dont la Politique Agricole Commune, les liens avec les organisations militaires, la politique régionale, ou encore l'attribution de postes au sein de la Commission européenne ${ }^{46}$.

42. Ces derniers mots ne furent pas diffusés par la radio irlandaise; on informa par la suite l'ambassadeur que la bande magnétique était trop courte pour les enregistrer...

43. AEAD : Europe, Irlande, vol. 54, f 75 : «Fiches d'ambassade », 1966.

44. Assemblée nationale, Rapport d'information, n 885, 12 nov. 1969, p. 28.

45. AEAD : Europe, Irlande, vol. 51 : Jurgensen, " Note pour le ministre », 5 sept. 1970.

46. AEAD : Europe, Irlande, carton 3356 : «Audience de Monsieur Jack Lynch, le 18 octobre 1972 ». 


\section{圈 L'Irlande du Nord}

Un autre enjeu permet de mesurer l'ampleur du rapprochement franco-irlandais : l'Irlande du Nord. Après de la Seconde Guerre mondiale, la France avait toujours refusé son soutien à Dublin sur la question de la partition, jugée beaucoup trop sensible vis-à-vis de l'alliance franco-britannique et donc de l'OTAN. Mais à partir de la fin des années soixante, le renouveau de l'amitié entre Paris et Dublin et le début du conflit en Irlande du Nord amena une inflexion notable dans la position des autorités françaises.

Dès le mois d'août 1969, lorsque le gouvernement irlandais voulut soulever la question de l'Irlande du Nord devant le Conseil de sécurité de l'ONU, la France apporta une assistance discrète mais fort appréciée de Dublin ${ }^{47}$. La politique menée par le Premier ministre irlandais, Jack Lynch, était perçue de manière positive par les autorités françaises, qui estimaient que ce "partisan de la conciliation et du dialogue " n'avait pas " cessé d'affirmer sur le ton de la plus grande modération et dans un esprit à la fois réaliste et libéral, l'objectif de l'unité nationale ${ }^{48}$ ". Non seulement il n'avait pas dévié de la voie de son prédécesseur, Seán Lemass, qui avait inauguré " une politique de rapprochement avec le gouvernement de Belfast ", mais au travers de circonstances difficiles il avait "affirmé avec courage son choix d'une politique de conciliation et de modération dans le délicat règlement de la question irlandaise ${ }^{49}$ ".

En 1970, lorsque le nouvel ambassadeur irlandais à Paris, Eamonn Kennedy, présenta ses lettres de créance au Président Pompidou et au ministre des Affaires étrangères, Maurice Schumann, il eut une discussion avec eux qui « porta presque exclusivement sur la situation en Irlande du $\operatorname{Nord}^{50} »$. Kennedy les trouva tous deux " très bien disposés à notre égard et compréhensifs ". Schumann lui promit de soulever la question de l'Irlande du Nord avec le ministre britannique des Affaires étrangères, ce que le diplomate irlandais jugea " très encourageant au vu de l'importance de la France quant au succès de la candidature britannique au Marché commun ".

En somme, l'approche traditionnelle des autorités françaises, fondée sur la non-ingérence dans les affaires intérieures d'un autre Etat, fut revue à la lumière de l'adhésion prochaine de la Grande-Bretagne à la CEE. Selon le Quai d'Orsay, il était désormais nécessaire d'encourager Londres à prendre des mesures en Irlande du Nord :

47. Christophe Gillissen, "Un “jeu subtil de discrétion et d'indiscrétion calculée” : la France et la question de l'Irlande du Nord à l'ONU ", Études Irlandaises, n 35-1 (printemps 2010), p. 39-53.

48. AEAD : Europe, Irlande, vol. 51 : « Note : M. John Lynch, Premier Ministre », 26 nov. 1970.

49. AEAD : Europe, Irlande, vol. $51:$ «Note : La situation politique intérieure en Irlande et la controverse autour du problème de l'unité ", 26 nov. 1970.

50. NA, DFA : Confidential report, 313/4/R : Kennedy, 15 juil. 1970. 
Jusqu'ici, nous avons considéré que l'affaire relevait de la souveraineté du gouvernement britannique, tout en souhaitant que celui-ci procède aux réformes nécessaires. Si une solution n'intervient pas rapidement, l'affaire d'Irlande du Nord risque cependant de nous concerner de plus près le jour où la Grande-Bretagne et l'Irlande seront toutes deux membres de la Communauté et participeront à la coopération politique ${ }^{51}$.

De fait, quelques mois plus tard, le gouvernement britannique lança une série de réformes majeures afin de trouver un règlement politique au conflit en Irlande du Nord : Edward Heath annonça la suspension du parlement nord-irlandais et le début de consultations intensives avec les partis de la région, sous l'égide de William Whitelaw, ministre des Affaires nord-irlandaises. Cette initiative ouvrit la voie aux accords de Sunningdale de décembre 1973, ainsi qu’à la formation d'un exécutif intercommunautaire en 1974.

Selon l'ambassadeur français à Dublin, deux facteurs expliquaient " la décision spectaculaire " du Premier ministre britannique ${ }^{52}$. Tout d'abord, une rencontre avec le chef du gouvernement irlandais lui avait permis de définir les bases d'un règlement acceptable de part et d'autre; mais dans le même temps, l'influence exercée par " les gouvernements amis de l'Irlande (France, Italie, Belgique, Danemark, États-Unis, Canada, Australie) » firent "prendre conscience à $\mathrm{M}$. Heath des réactions de l'opinion internationale ».

Dans le contexte d'une internationalisation du conflit, la dimension européenne n'était pas négligeable, puisque les candidatures britannique et irlandaise à la CEE faisaient de la situation en Irlande du Nord une affaire qui concernait désormais les Six, "en particulier la France et l'Italie » qui firent " pression sur le gouvernement britannique en vue d'aboutir à un règlement de l'affaire du Nord, avant que la Grande-Bretagne et l'Irlande ne deviennent membres de la Communauté ${ }^{53}$ ".

\section{Conclusion}

La transformation des perceptions françaises de l'Irlande et de sa demande d'adhésion à la CEE en l'espace de quelques années, ainsi que le rapprochement franco-irlandais, furent remarquables, tant par leur ampleur que par leur rapidité. Ils s'expliquent par nombre de facteurs, parmi lesquels on peut, en guise de conclusion, en souligner trois.

51. AEAD : Europe, Irlande, carton 3355 : « Note : la situation actuelle en Irlande du Nord », 15 oct. 1971.

52. AEAD : Europe, Irlande, carton 3355 : Harcourt, «Attitude du gouvernement irlandais à l'égard de l'initiative britannique en Irlande du Nord ", 30 mars 1972.

53. AEAD : Europe, Irlande, carton 3355, n 206/211: Harcourt, « Réactions irlandaises au plan Heath », 27 mars 1972. 
Tout d'abord, ils furent facilités par des changements profonds dans le contexte géopolitique. Le refus de Londres de participer à la fondation de la CEE en 1957 amoindrit considérablement la valeur de l'alliance franco-britannique aux yeux des décideurs français, qui optèrent dès lors pour un rapprochement avec l'Allemagne. Le projet d'une Europe indépendante plutôt qu'atlantiste mené par de Gaulle créa un espace où l'Irlande put se rapprocher de Paris et poser les bases d'une alliance franco-irlandaise au sein de la CEE.

Par ailleurs, la candidature irlandaise à l'organisation européenne entraina de nombreuses rencontres bilatérales avec les officiels français, ce qui permit à ces derniers de mieux connaitre leurs homologues irlandais et de forger des relations personnelles fondées sur une sympathie et une confiance mutuelles ${ }^{54}$. En 1961, Paris cherchait à garder ses distances vis-à-vis d'un pays encore mal connu, mais dès 1964 les autorités françaises comprirent tout l'intérêt qu'elles avaient à développer leurs liens avec Dublin dans la perspective d'un élargissement de la CEE, grâce aux visites et entretiens franco-irlandais.

Enfin, il faut insister sur l'habileté et la persévérance de la diplomatie irlandaise, confrontée à un défi de taille lorsque Dublin se rendit compte en 1961 que la France était opposée à sa candidature. Si les changements géopolitiques créèrent une ouverture pour l'Irlande, son accession à la CEE n'était pas gagnée d'avance, loin s'en faut. Il lui fallut bien du talent et de la patience pour persuader les autorités françaises que non seulement l'Irlande ne serait pas un satellite de la GrandeBretagne, mais que Paris et Dublin avaient des intérêts communs qui seraient mieux défendus s'ils agissaient de concert au sein de la CEE.

54. Joseph T. Carroll, "General de Gaulle and Ireland's EEC application », in P. Joannon (dir.), De Gaulle and Ireland, op. cit., p. 81-97. 